\title{
Stratospheric Temperature Profiles from Limb Radiance Measurements
}

\author{
Thomas B. McKee and Stephen K. Cox \\ Dept. of Atmospheric Science, Colorado State University, Fort Collins 80521
}

(Manuscript received 19 October 1972, in revised form 22 March 1973)

\section{ABSTRACT}

\begin{abstract}
Stratospheric temperature profiles have been inferred from limb radiance data. The limb radiance observations were made from an Aerobee rocket flown on 7 February 1970 from White Sands Missile Range, N. M. The inferred temperatures were similar to those available from rocket sounding data. Horizontal variations of temperature at the 10 -mb surface agreed with radiosonde data; however, the inferred temperatures were systematically warmer at $10 \mathrm{mb}$ with larger increases of temperature from 30 to $10 \mathrm{mb}$ than comparative radiosonde data. Two of the inferred temperature profiles provide an excellent example in which two measurements are sufficiently alike to separate real vertical temperature structure from effects of random radiance noise. Vertical resolution was about $2 \mathrm{~km}$ and the largest lapse noted was near dry adiabatic.
\end{abstract}

\section{Introduction}

One approach for remotely probing the stratosphere is to view the earth's limb with a radiometer with a small optical field of view from a rocket or orbiting satellite. Such an instrument would instantaneously receive radiation from primarily a rather thin layer of the atmosphere. Advantages of viewing the atmosphere tangentially are the availability of larger optical depths of gases at low pressures and small mixing ratios and enhanced vertical resolution. One disadvantage is that the spherical nature of the atmosphere must be considered. Techniques to infer temperature as a function of pressure from limb radiance data have been developed by McKee (1970) and Gille and House (1971).

The present paper presents a brief description of the technique to infer stratospheric temperature as a function of pressure from limb radiance data (McKee, 1970) using thermal emission from carbon dioxide in the $15-\mu \mathrm{m}$ spectral region. The technique is applied to a set of experimental radiance data (Walker, 1971) ${ }^{1}$ and the temperatures are compared with available radiosonde and rocketsonde data.

\section{Limb radiance}

Theoretical computations of limb radiance are made from numerical evaluation of the integral form of the equation of radiative transfer:

$$
\lambda=\int N_{\nu}\left(s_{0}\right) \tau_{\nu}\left(s_{0}\right) d \nu-\iint J_{\nu} \frac{\partial \tau_{\nu}}{\partial s} d s d \nu
$$

\footnotetext{
1 Private communication.
}

in which $N$ is the radiance, $N_{v}$ the spectral radiance, $\tau_{\nu}$ the spectral transmittance, $J_{\nu}$ the source function, and $s$ the distance along the path of integration. The first term is a boundary term of the radiance emitted at a boundary depleted by the extinction through the gas. The second term is the contribution to radiance from the gas along the path.

The geometry for which Eq. (1) must be evaluated for limb radiance is shown in Fig. 1. The atmosphere is assumed to be spherically stratified. A line of sight from an observer through the atmosphere is uniquely specified by the altitude where the radius vector from the center of the earth forms a right angle with the line of sight. This altitude is called the tangent height and is the minimum altitude from the surface along the line of sight. Total atmospheric pressure is also a unique characteristic of the tangent point and is called tangent pressure. A limb radiance profile is defined as the radiance from different lines of sight as a function of tangent pressure.

For lines of sight with tangent pressures in the stratosphere where clouds, solid boundaries, or radiation sources beyond the atmosphere are not normally present, the first term in Eq. (1) is zero; this reduces the transfer equation to

$$
N=-\iint J_{\nu} d \tau_{\nu} d \nu
$$

In the earth's stratosphere thermodynamic equilibrium prevails (Goody, 1964) and, neglecting scattering, the source function becomes the Planck function

$$
J_{v}=N_{B v}=\frac{C_{1} \nu^{3}}{\exp \left(C_{2} \nu / T\right)-1}
$$




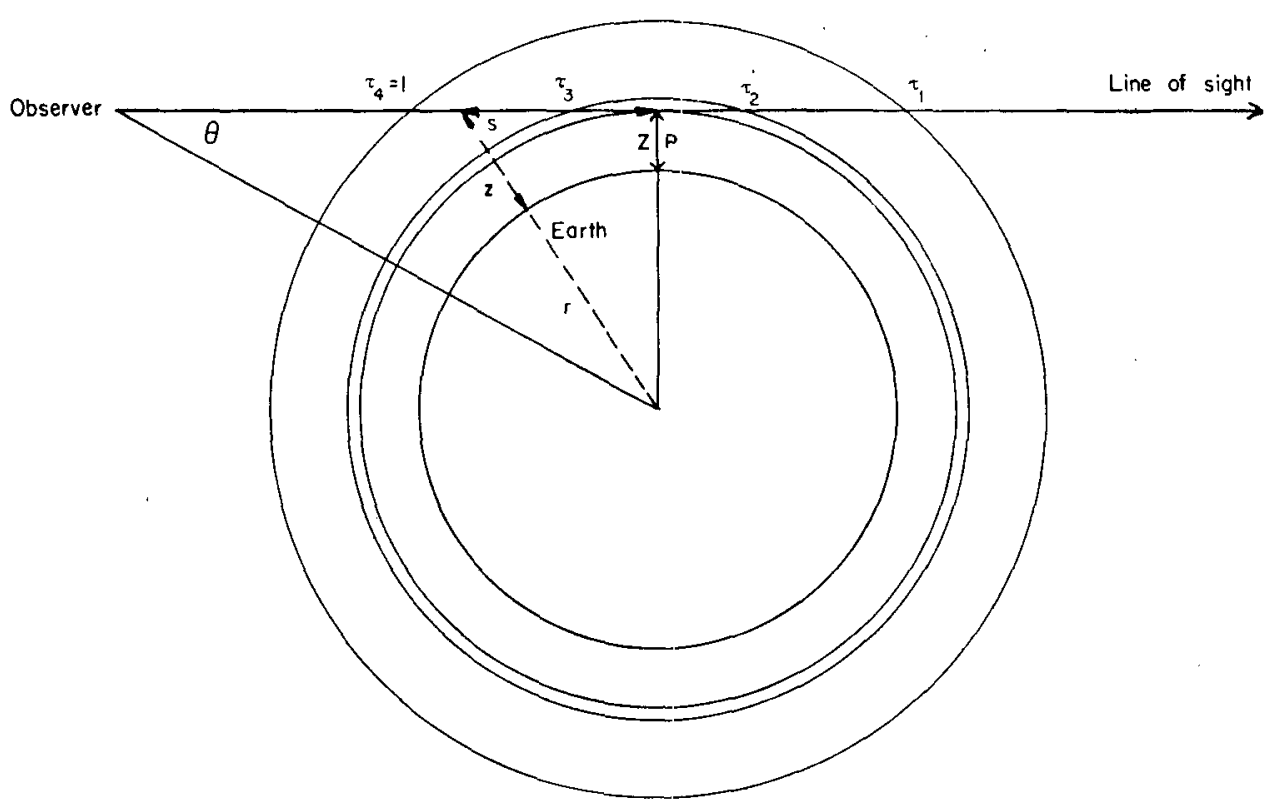

FIG. 1. Limb geometry.

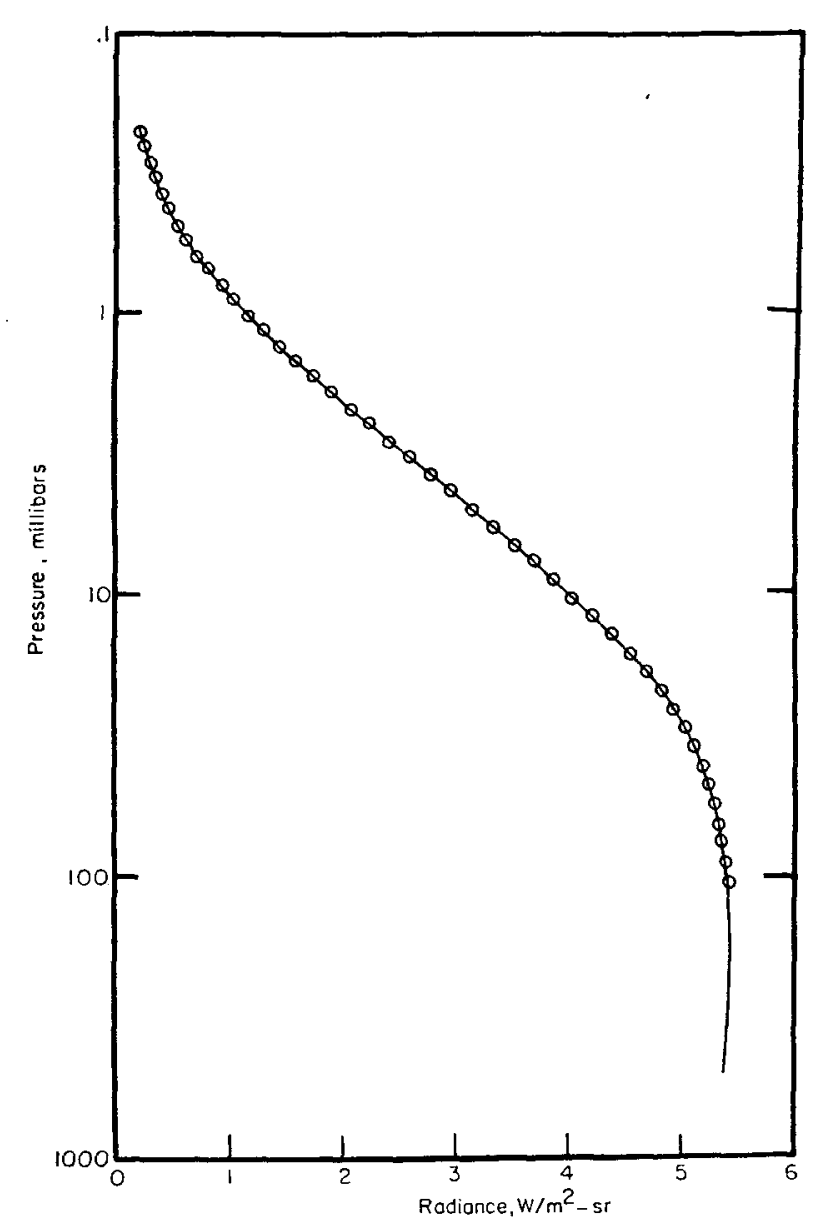

FIG. 2. Limb radiance profile for 615 to $715 \mathrm{~cm}^{-1}$ for the 1962 U. S. Standard Atmosphere. with temperature $T$, wavenumber $\nu$, and the first and second radiation constants $C_{1}$ and $C_{2}$.

Evaluation of Eq. (2) requires a knowledge of $\tau_{\nu}$ as well as the source function. The transmittance calculation technique reported by Bates et al. (1967) based on the quasi-random model results has been used in the present work. Transmittance is weakly clependent on temperature, strongly dependent on pressure, and strongly dependent on the amount of a.bsorbing gas. Thus, the meteorological variables of temperature, pressure and mixing ratio must be specified. The mixing ratio for $\mathrm{CO}_{2}$ was assumed constant throughout the stratosphere with a value of $314 \mathrm{ppm}$.

A limb radiance profile computed for the 615 to $715 \mathrm{~cm}^{-1}$ spectral interval due to thermal emission of $\mathrm{CO}_{2}$ is shown in Fig. 2. Temperature data as a function of pressure were from the $U$. S. Standard Atmosphere (1962).

\section{Inference of temperature}

Inference of temperature is the problem of starting with the integral value of $N(P)$ and deducing the temperature influence of the integrand. A derivation of the technique used in the present work was given by McKee (1970).

The inference begins with a small value of radiance for a small tangent pressure and proceeds to larger values of tangent pressure. For the first radiance value used, a temperature and pressure of the tangent point are assumed to be $T_{0}$ and $P_{0}$. The atmosphere at smaller pressure is assumed to have a constant 


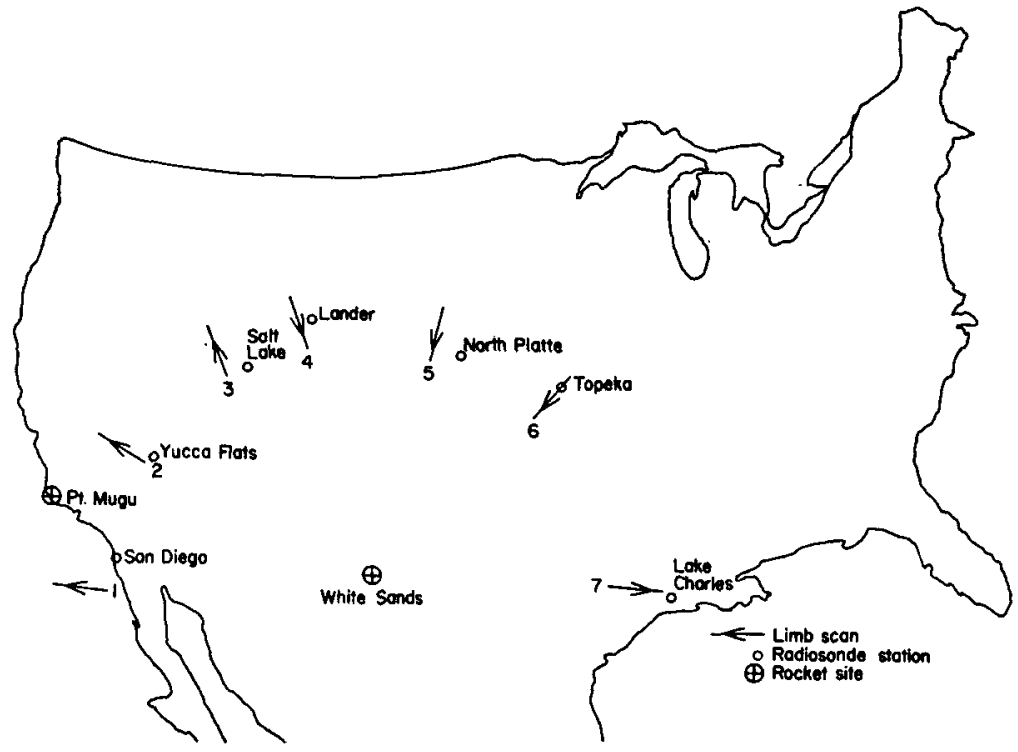

FIG. 3. Geographic locations of limb radiance scans.

lapse rate with height, $\gamma$, and a temperature given by

$$
T=T_{0}\left(\frac{P}{P_{0}}\right)^{-R \gamma / v}
$$

A theoretical radiance $N_{t}$ is calculated from Eq. (2) and compared with a measured radiance $N_{m}$, which results in a radiance residual of

$$
\Delta N=N_{m}-N_{t} .
$$

The temperature $T_{0}$ is changed in an iterative process until $\Delta N\left(P_{0}\right)$ is made arbitrarily small. The assumed value of $P_{0}$ is not changed.

A new radiance value is selected and the change in tangent height, $\Delta Z$, between the previous and present radiance values is determined from the vertical scan rate and time between observations. The tangent pressure of the new radiance value, $P_{i}$, is related to the previous tangent pressure, $P_{i-1}$, and the average temperature of the new layer, $T_{a}$, through the hydrostatic expression by

$$
P_{i}=P_{i-1} \exp \left(g \Delta Z / R T_{a}\right) .
$$

As previously, a temperature is assumed and a radiance residual is calculated. An iteration is performed on $T_{a}$ until the radiance residual is made small; however, the new tangent pressure is changed each time $T_{a}$ is changed to insure the new layer is in hydrostatic balance. The inference technique then proceeds in a similar manner including additional successive layers of the atmosphere.

\section{Inference of temperature from experimental radiance data}

Several measurements of limb radiance profiles have been reported in the past few years by Walker et al.
(1966), McKee et al. (1968), Whitman et al. (1968) Girard (1970) and Walker (loc. cit.). All of these experiments included measurements in the $15-\mu \mathrm{m}$ region of $\mathrm{CO}_{2}$ emission. However, only the measurements of Walker et al. (loc. cit.) used a radiometer with sufficient signal-to-noise ratio to provide radiance data suitable for an inference application. This section describes the experimental limb radiance profiles, the meteorological data available near the time and location of the radiance measurements, the inference of temperature from the limb radiance data, and the comparison of inferred temperature with the meteorological data.

\section{a. Experimental radiance data}

An infrared radiometer was included in the instrumentation on an Aerobee rocket launched from White Sands Missile Range, N. M., at approximately 0530 GMT on 7 February 1970. Apogee for the probe was $145 \mathrm{~km}$. The payload was erected to near the local vertical and was spinning about the vertical axis while an oscillating mirror in the radiometer scanned the instrument line of sight up and down across the earth's limb. Limb crossings of the line of sight are contained in a circle around the sub-vehicle point. Geographical locations of seven actual limb crossings are illustrated in Fig. 3. For a fixed rocket vehicle altitude, which is nearly the condition during one scan, the tangent point changes location for different lines of sight; the arrows in Fig. 3 indicate the direction of movement. A scan from space to earth causes the tangent point to move away from the observer such as in scan numbers 1, 2, 3 and 7 . The end points of each scan in Fig. 3 mark approximate limits where radiance data were measured. Radiance data from each of 


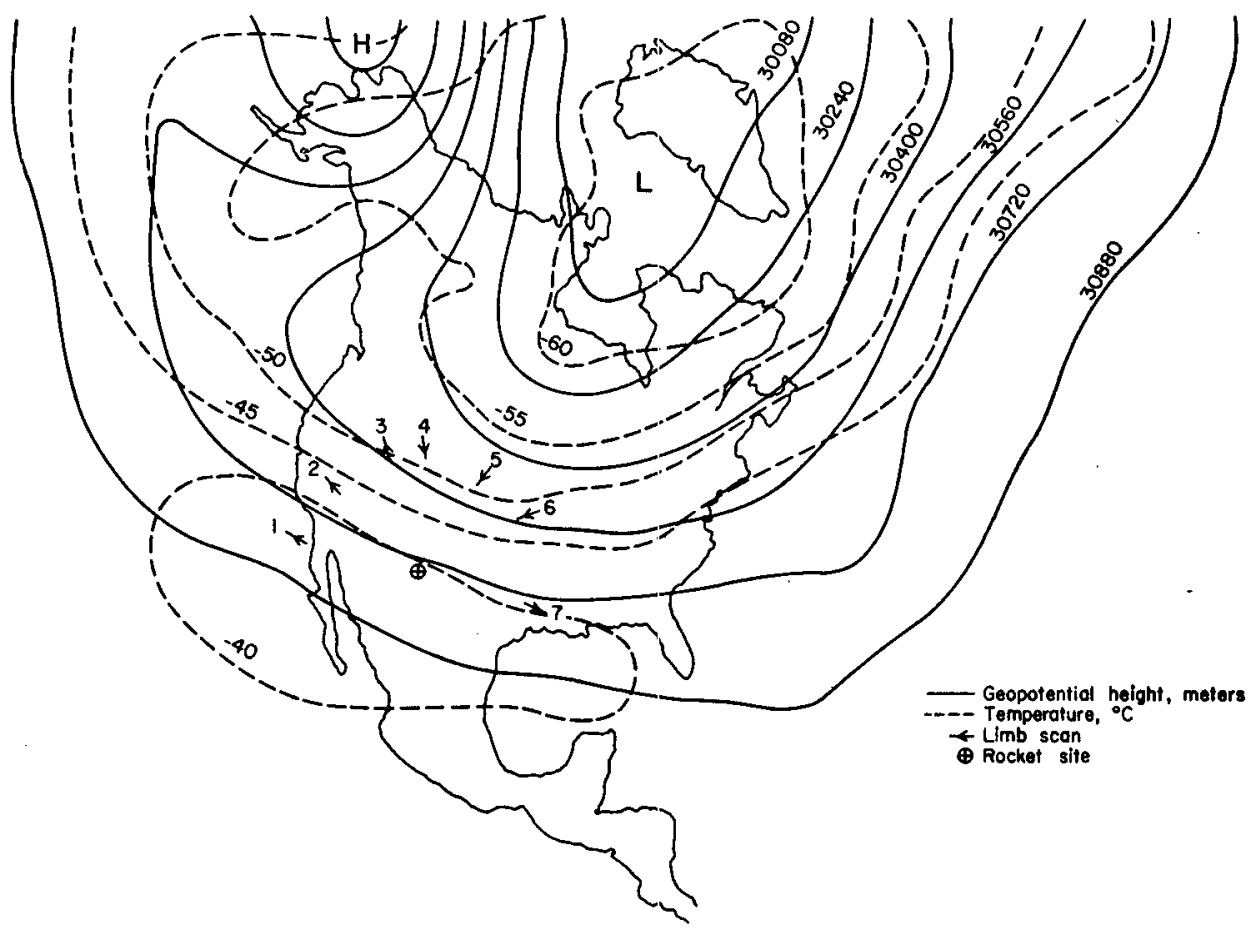

FIG. 4. Stratospheric analysis at $10 \mathrm{mb}$ fcr 7 February 1970 (NOAA, 1970).

these seven scans have been provided prior to publication by Walker et al. (loc. cit.). These radiance data were observed with an instrument which had a spectral response with $50 \%$ values from about 645 to $707 \mathrm{~cm}^{-1}$. An estimate of the standard deviation of the noise level for the radiance data is $0.027 \mathrm{~W} \mathrm{~m}^{-2} \mathrm{sr}^{-1}$. Measured radiance data were available at tangent height spacings of about $0.25 \mathrm{~km}$. A seven-point average was taken centered on every fourth data point which yielded smoothed data with about $1-\mathrm{km}$ spacing representing a $1.5 \mathrm{~km}$ vertical averaging. The linear smoothing resulted in an estimated standard deviation due to noise of $0.01 \mathrm{~W} \mathrm{~m}^{-2} \mathrm{sr}^{-1}$.

In the experiment radiance was measured as a function of tangent height. The information needed for the present inference application is the change of tangent height between radiance values which is defined in Eq. (6). Consequently, only the change of tangent height has been used.

TABLE 1. Radiosonde stations used with limb radiance scans.

\begin{tabular}{cl}
\hline Scan & Radiosonde station \\
\hline 1 & San Diego, Calif. \\
2 & Yucca Flats, Nev. \\
3 & Salt Lake City, Utah \\
4 & Lander, Wyo. \\
5 & North Platte, Nebr. \\
6 & Topeka, Kan. \\
7 & Lake Charles, La. \\
\hline
\end{tabular}

\section{b. Meleorological data}

The meteorological temperature data available for comparison with inferred temperature data include the regular 0000 and 1200 GMT soundings taken at each station shown in Fig. 3 and a few rocket soundings. Data from the $500-\mathrm{mb}$ analysis show a longwave or semipermanent trough in the eastern half of the United States which did not change appreciably from 1200 GMT 6 February to 1200 GMT 7 February. This longwave trough is also apparent in the analyses at $100,50,30$ and $10 \mathrm{mb}$. The $10-\mathrm{mb}$ analysis (National Oceanic and Atmospheric Administration, 1970) is shown in Fig. 4. At $50 \mathrm{mb}$ the normal horizontal temperature gradient in the troposphere with colder temperatures to the north has been replaced with warmer temperatures to the north. By $10 \mathrm{mb}$ the gradient with colder temperatures to the north has been reestablished and should continue to the stratopause. Geographical location of the seven limb scans are also depicted on the $10-\mathrm{mb}$ analysis.

Radiosonde data at 0000 GMT 7 February 1970 were taken $5.5 \mathrm{hr}$ prior to the radiance measurements and the 1200 GMT data were taken $6.5 \mathrm{hr}$ after the radiance measurements. Only small temperature differences were observed between the two radiosonde measurements and no significant advection of temperature is expected from the analysis. Consequently, the radiosonde data were considered to represent the a.tmosphere from 50 to $10 \mathrm{mb}$ at the time of the radiance measurements. The radiosonde stations used 
to provide the temperature as a function of pressure up to $10 \mathrm{mb}$ for each limb scan are given in Table 1 .

Rocketsonde data reported by World Data Center A (1970) have been obtained for Pt. Mugu, Calif., and White Sands, N. M., for the times nearest to that of the radiance measurements. Pt. Mugu is geographically closer to the location of the radiance measurements. In the time period 5-11 February 1970 only two soundings were made which included a temperature measurement, i.e., those at 2106 GMT 5 February and 2202 GMT 11 February. These were both daytime soundings. General similarity of the two rocket soundings indicated no large changes had taken place in the atmosphere in the intervening period. A series of rocketsondes were launched at White Sands on 9 February with five soundings taken between 1706 and 2030 GMT. An earlier sounding was taken on February 6 at 1900 GMT. Again all measurements were taken in the daytime. Variations in measured temperature on 9 February were greater than the change from 6 to 9 February.

\section{c. Discussion of inferred temperature structure}

The derivation of the inference technique left the tangent pressure of the initial level an arbitrary constant. The temperature solution obtained is sensitive to the value of $P_{0}$ assumed. The method used for determining the initial pressure was to match the inferred temperature structure with the radiosonde measurements at the lower boundary (Gille and House, 1971). In the present example radiosonde data were available close in space and time for each limb scan so they were used to determine the initial pressure. Both temperature and lapse rate from $10 \mathrm{mb}$ to about $40 \mathrm{mb}$ were used to define the best fit at the lower boundary.

Sensitivity of the inference technique to radiance errors is shown in Fig. 5. A radiance error of 0.01 $\mathrm{W} \mathrm{m} \mathrm{m}^{-2} \mathrm{sr}^{-1}$ introduced in the tangent layer would cause the temperature error shown. At $0.6 \mathrm{mb}$ where the atmosphere is quite transparent and radiances are small the error was $3.4 \mathrm{~K}$. At larger pressures the radiance increases and the sensitivity decreases to less than $1 \mathrm{~K}$. For tangent pressures $>17 \mathrm{mb}$ the atmosphere tends toward opacity and the error again becomes larger than $1 \mathrm{~K}$.

The temperature structure inferred from the seven limb radiance scans is shown in Fig. 6. Results of choosing the initial pressure for the inference so that the temperature structure matches the radiosonde data of the lower boundary are illustrated. Inferred temperatures are warmer than radiosonde temperatures at $10 \mathrm{mb}$ but agree within the noise in the inferred data by $30 \mathrm{mb}$. Muench (1971) has shown that radiosonde temperature measurements tend to have a systematic error which increases with height above $25 \mathrm{~km}$ causing observed temperatures to be too low. Fig. 6

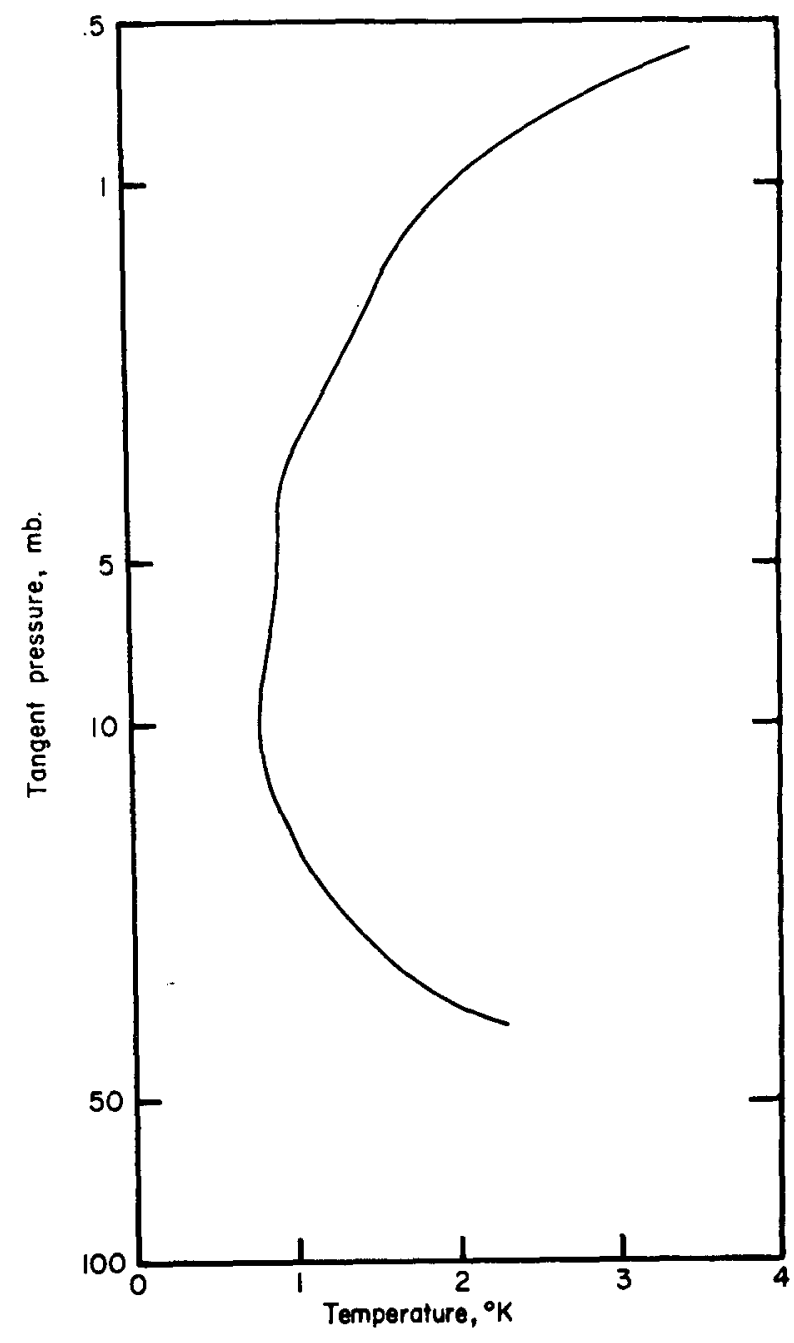

FIG. 5. Temperature error for a radiance error of $0.01 \mathrm{~W} \mathrm{~m}^{-2} \mathrm{sr}^{-1}$.

indicates that the discrepancies between inferred and radiosonde temperatures are of the same nature as Muench (1971) has described. Horizontal temperature gradients which were present also tend to produce a larger lapse rate at $10 \mathrm{mb}$; however, the temperature gradients evident in Fig. 4 are not large enough to account for all of the difference noted in Fig. 6 (McKee, 1972).

Much of the detailed structure evident in the inferred temperatures is due to the random radiance noise of the measuring instrument. Separation of actual temperature structure from effects of random radiance noise cannot be accomplished with just one inferred temperature profile. Comparison of two of the inferred temperature profiles which are close together geographically affords an opportunity to separate structure from noise since noise is not repeatable. Scans 3 and 4 are about $400 \mathrm{~km}$ apart. The vertical temperature structure from 0.8 to $3 \mathrm{mb}$ is 


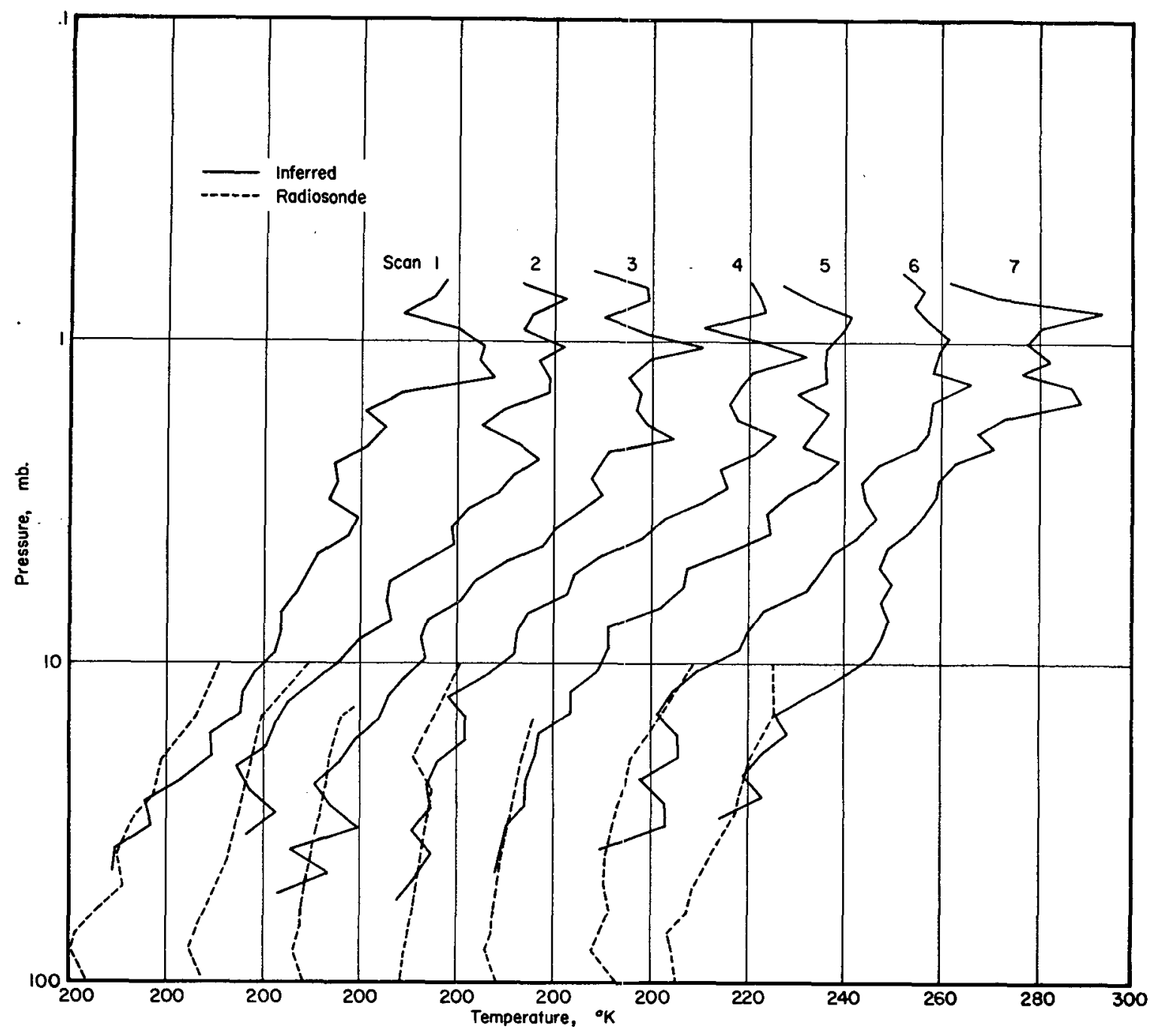

FIG. 6. Inferred temperature structure.

nearly identical and is considered real. Thirteen radiance values were used between these pressures and the probability of random errors being so similar is extremely small. In fact, the probability that the two random errors would have the same sign thirteen times in a row is $\left(\frac{1}{2}\right)^{13}$. The structure at pressures $>10 \mathrm{mb}$ appears to be due to random noise as very little correlation is evident. The large decrease in temperature near $1 \mathrm{mb}$ amounts to $20 \mathrm{~K}$; the lapse rate of $11 \mathrm{~K} \mathrm{~km}^{-1}$ is just slightly greater than dry adiabatic. Detailed structure in scans 3 and 4 indicate a vertical resolution of about $2 \mathrm{~km}$ for the inferred data. Vertical resolution will diminish at larger pressures as the atmosphere becomes opaque.

In Fig. 6 the lapse rates from 10 to $2 \mathrm{mb}$ are quite similar for scans 2, 3, 4, 5 and 6 but scans 1 and 7 are noticeably different. Examination of the $10-\mathrm{mb}$ analysis in Fig. 4 reveals that scans 1 and 7 are in similar locations relative to the large trough but are quite different from scans 3 and 4 . Temperatures and lapse rates in scans 1 and 7 are similar throughout the profile. The temperature of scans 1 and 7 at $10-\mathrm{mb}$ are the warmest of all scans which is consistent with the $10 \mathrm{mb}$ analysis of Fig. 4.

Scans 1 and 2 are located on opposite sides of the rocket site at Pt. Mugu, Calif. The 5 and 11 February rocket soundings are compared with temperatures from these scans in Fig. 7. The inferred profilescertainly appear reasonable for locations on either side of the rocket site as the rocket data are between the inferred temperatures at most pressures. Differences between the average of the two rocket soundings and the average of the two scans yield an rms value of about $3 \mathrm{~K}$. Diurnal temperature change is not evident even though the rocket data are near mid-day and the inferred data at night. Diurnal change should be the largest in the vicinity of $1 \mathrm{mb}$ but a summary of diurnal effects by Gille (1968) indicates considerable uncer- 


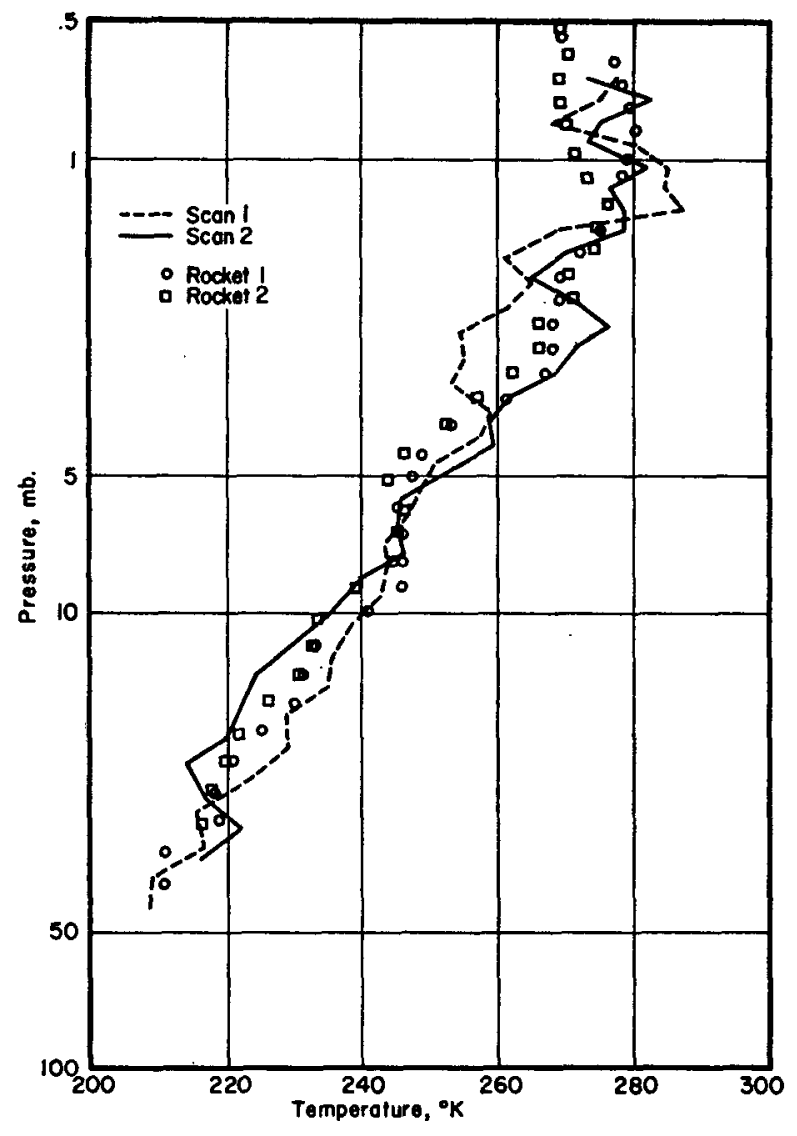

FIG. 7. Comparison of inferred temperatures with rocket soundng temperature data obtained on 5 February 1970 (circles) and 11 February 1970 (squares).

tainty about the local time of temperature maximum which varied from noon to early evening for theory and from noon to near midnight for observations. Rocket data from White Sands also have an average lapse rate from 10 to $2 \mathrm{mb}$ more like scans 1 and 7 than any of the others. One of the four White Sands soundings has a distinct warm bulge at pressure a little smaller than $10 \mathrm{mb}$ similar to that of scan 7 .

\section{Concluding remarks}

A technique to infer stratospheric temperature from limb radiance profiles due to emission by $\mathrm{CO}_{2}$ has been described. The technique was applied to a set of seven limb radiance profiles measured in a spectral interval of 645 to $707 \mathrm{~cm}^{-1}$.

Inferred temperatures are similar to those from available rocket sounding data. Horizontal variations of temperature at $10 \mathrm{mb}$ agree with radiosonde data; however, the inferred temperatures were systemati- cally warmer at $10 \mathrm{mb}$ with larger increases of temperature from 30 to $10 \mathrm{mb}$ than comparative radiosonde data. Two of the inferred temperature profiles provide an excellent example in which two measurements are sufficiently alike to separate real vertical temperature structure from the effects of random radiance noise. Vertical resolution was about $2 \mathrm{~km}$ and the largest lapse rate noted was near dry adiabatic.

Acknowledgments. This research was supported by the Atmospheric Sciences Section, National Science Foundation, under Grant GA-18783. The authors wish to thank Dr. Russell Walker of the Air Force Cambridge Research Laboratories for providing his experimental data prior to publication.

\section{REFERENCES}

Bates, Jerry C., David S. Hansen, Fred B. House, Robert O'B. Carpenter and John C. Gille, 1967: The synthesis of $15 \mu$ infrared horizon radiance profiles from meteorological data inputs. NASA CR-724.

Gille, J. C., 1968: On the possibility of estimating diurnal temperature variation at the stratopause from horizon radiance profiles. J. Geophys. Res., 73, 1863-1868.

- - and Frederick House, 1971: On the inversion of limb radiance measurements: I. Temperature and thickness. $J$. Atmos. Sci., 28, 1427-1442.

Girard, A., 1970: Profiles experimentaux de l'horizon. $A p p l . O p l$, 9, 903-912.

Goody, R. M., 1964: Atmospheric Radiation. I. Theoretical Basis. London, Oxford University Press, 426 pp.

McKee, Thomas B, 1970: Inference of temperature and water vapor structure in the stratosphere from limb radiance profiles. NASA-TM-X-1943.

- - 1972: Inference of stratospheric temperature and water vapor from limb radiance profiles. Atmos. Sci. Paper No. 178, Colorado State University, Fort Collins, $111 \mathrm{pp.}$

_- Ruth I. Whitman and Richard E. Davis, 1968: Infrared horizon profiles for summer conditions from project scanner. NASA-TN-D-4741.

Muench, H. Stuart, 1971: Temperature measurements in the 30 to 40 kilometer region. Mon. Wea. Rev., 99, 158-161.

National Oceanic and Atmospheric Administration, 1970: Daily series, synoptic weather maps: Part II. Northern Hemispheric Data Tabulations, February, 1970. Asheville, N. C., National Climatic Center.

U. S. Standard Atmosphere (1962): Washington, D. C., Gov't. Printing Office.

Walker, R. G., C. V. Cunniff and A. P. D'agati, 1966: Measurement of the infrared horizon of the earth. AFCRL-66631, Environmental Research Paper No. 223, Air Force Cambridge Research Laboratories, Bedford, Mass.

Whitman, Ruth I., Thomas B. McKee and Richard E. Davis, 1968: Infrared horizon profiles for winter conditions from project scanner. NASA-TN-D-4905.

World Data Center A, 1970: Data report high altitude meteorological data. Vol. 7, No. 2, Asheville, N. C., National Weather Records Center. 